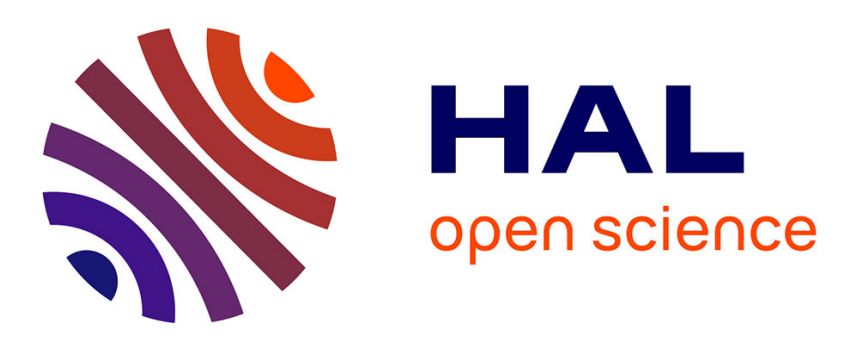

\title{
Investigations on OFDM Signal for Range Ambiguity Suppression in SAR Configuration
}

\author{
Vishal V. Riché, Stéphane Meric, Jean-Yves Baudais, Eric Pottier
}

\section{To cite this version:}

Vishal V. Riché, Stéphane Meric, Jean-Yves Baudais, Eric Pottier. Investigations on OFDM Signal for Range Ambiguity Suppression in SAR Configuration. IEEE Transactions on Geoscience and Remote Sensing, 2014, 52 (7), pp.4194-4197. 10.1109/TGRS.2013.2280190 . hal-00831837

\section{HAL Id: hal-00831837 https://hal.science/hal-00831837}

Submitted on 18 Sep 2014

HAL is a multi-disciplinary open access archive for the deposit and dissemination of scientific research documents, whether they are published or not. The documents may come from teaching and research institutions in France or abroad, or from public or private research centers.
L'archive ouverte pluridisciplinaire HAL, est destinée au dépôt et à la diffusion de documents scientifiques de niveau recherche, publiés ou non, émanant des établissements d'enseignement et de recherche français ou étrangers, des laboratoires publics ou privés. 


\title{
Investigations on OFDM Signal for Range Ambiguity Suppression in SAR Configuration
}

\author{
Vishal Riché, Stéphane Méric, Member, IEEE, Jean-Yves Baudais, and Éric Pottier, Fellow, IEEE
}

\begin{abstract}
This paper presents an opportunity to cancel range ambiguities in synthetic aperture radar (SAR) configuration. One of the limitations of SAR systems is the range ambiguity phenomenon that appears with long delayed echoes. The reflected signal corresponding to one pulse is detected when the radar has already transmitted the next pulse. Thus, this signal is considered as an echo from the next pulse. This paper investigates the opportunity of coding the transmitted pulses using an orthogonal frequency-division multiplexing pulse. The results show that coded-OFDM signals outperform conventional chirp signal and make it possible to relax constraints placed upon the pulse repetition frequency.
\end{abstract}

Index Terms-Image quality parameters, orthogonal frequency-division multiplexing (OFDM) signals, range ambiguity, synthetic aperture radar (SAR) processing.

\section{INTRODUCTION}

$\mathbf{F}$ OR remote sensing applications in the radar domain, specific signal processing is necessary to obtain high resolution for radar images. This high resolution is essential in detection and imaging processing, and it is provided by using synthetic aperture radar (SAR) processing. For strip map SARs, one of the major interferences is the arrival of an unwanted echo after the transmission of a new pulse. Thus, range ambiguities appear if the current transmitted pulse does not contribute to the backscattered signal that is received after the transmission of a new pulse. These late echoes can appear when they are located after the maximum unambiguous range defined by the radar system. These kinds of echoes appear as shadows and can be misinterpreted. Thus, removal of this interference is an important matter for the SAR. The fundamental and theoretical analyses on ambiguities have been already achieved [1], and the ambiguity management becomes an important issue as the value of pulse repetition frequency (PRF) rises, whereas reasonable swath widths are required [2]. The use of a low PRF makes it possible to mitigate range ambiguities but induces Doppler

Manuscript received June 30, 2012; revised December 21, 2012; accepted February 1, 2013

V. Riché was with the SAPHIR Team, Institute of Electronics and Telecommunications of Rennes, CNRS UMR 6164, 35042 Rennes, France. He is now with the Fraunhoffer Institut, 53343 Wachtberg, Germany (e-mail: vishal. riche@laposte.net).

S. Méric and É. Pottier are with the SAPHIR Team, Institute of Electronics and Telecommunications of Rennes, CNRS UMR 6164, 35042 Rennes, France (e-mail: stephane.meric@insa-rennes.fr).

J.-Y. Baudais is with the French National Centre for Scientific Research (CNRS), Institute of Electronics and Telecommunications of Rennes (IETR), CNRS UMR 6164, 35043 Rennes, France.

Color versions of one or more of the figures in this paper are available online at http://ieeexplore.ieee.org.

Digital Object Identifier 10.1109/TGRS.2013.2280190 ambiguities that degrade the azimuth resolution [3]. In order to suppress the ambiguity without degrading the resolution, several ambiguity suppression techniques have been proposed. The use of alternating up and down chirp modulation is often presented to resolve the range ambiguity problem [4]. Another technique is based on the azimuth phase coding in order to eliminate the ambiguities [5]. One can also find a pulse block coding technique that makes it possible to detect false targets due to the range ambiguity while using matched filtering operations [6]. In a very recent paper [7], the issue of the orthogonal frequencydivision multiplexing (OFDM) coding for range ambiguities mitigation has been described taking into consideration the range-ambiguity-to-signal-ratio value. As in [7], we propose to use the OFDM coding technique to improve the range ambiguity rejection. Several works deal with the ambiguity function to design the OFDM signal. In [8]-[10], the authors propose to enhance the performance of detection applications by providing good range-Doppler properties through OFDM coding. In this paper, we propose to code the OFDM signals to optimize different imaging quality parameters, i.e., the peak sidelobe ratio (PSLR), the integrated sidelobe ratio (ISLR), and the interambiguity function. This paper is organized as follows. Section II describes the issue of the range ambiguity with OFDM signals. In Section III, we propose some simulation results demonstrating the efficiency of coded-OFDM signals for range ambiguity suppression.

\section{RANGE AMbiguity SUPPRESSION WITH OFDM SIGNALS}

\section{A. Range Ambiguity}

In radar systems, the presence of ambiguities (range and azimuth) is well known and largely studied [11]. Thus, there are contradictions between increase in PRF and the decrease in PRF. On the one hand, the increase in PRF induces azimuth ambiguities while reducing the range ambiguities; on the other hand, the decrease in PRF induces range ambiguities while reducing the azimuth ambiguities. In this paper, we propose to consider the opportunity to increase the PRF without raising the range ambiguity. We present in Fig. 1 the slant geometry in SAR configuration where $\mathrm{PRF}_{0}$ is the pulse repetition, which is set up to have the maximum swath width without ambiguities by taking into account the height $H$ of the radar, near incidence angle $\theta_{n}$, and far incidence angle $\theta_{f}$. In our case, we basically consider that the radar system transmits two pulses $s_{a}(t)$ and $s_{b}(t)$ one after the other. Thus, to reject the range ambiguity that can occur if we consider PRF $>\mathrm{PRF}_{0}$ (see Fig. 1), we develop a process capable of extracting the ambiguous response of the 


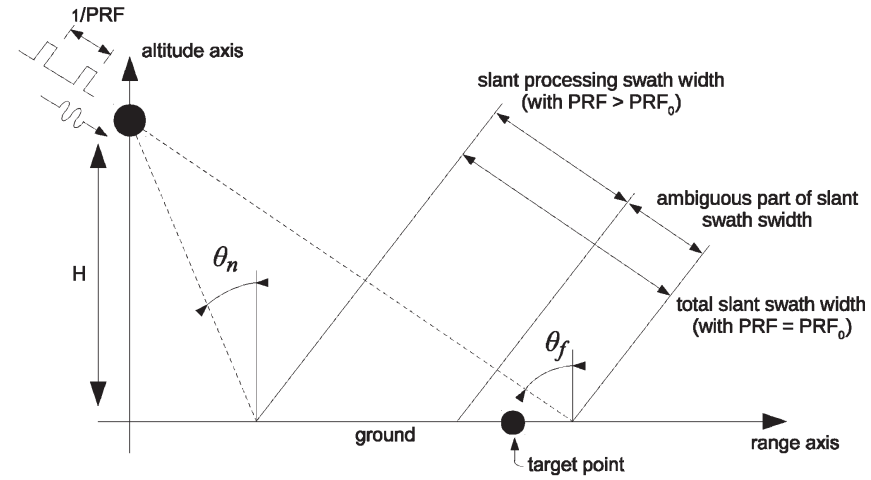

Fig. 1. SAR imaging slant geometry.

point target, which is visible in the nonambiguous radar image, as described in [12].

\section{B. OFDM Signal}

The principle of OFDM communication consists in the simultaneous transmission of multiple orthogonal subcarriers [13]. The analytical expression without cyclic prefix of one OFDM symbol composed of $N$ subcarriers $f_{i}$, transmitting the $N$ complex data symbols $a_{i}$ at the carrier frequency $f_{c}$, is as follows:

$$
s_{a}(t)=\exp \left(j 2 \pi f_{c} t\right) \sum_{i=1}^{N} p(t) a_{i} \exp \left(j 2 \pi f_{i} t\right)
$$

where $p(t)$ is the shaping function. In our case, we use the rectangular shaping function. The length of the OFDM symbol is $T_{s}=N / B_{r}$, where $B_{r}$ is the total bandwidth of the transmitted signal. The subcarrier $f_{i}$ is $(i-(N+1) / 2) / T_{s}$ with $N$ odd. To generate two nonoverlapping OFDM signals, we choose coefficient $a_{i} \in\{0 ; 1\}$, where $a_{i}=1$ means that subcarrier $f_{i}$ is present and $a_{i}=0$ otherwise. If $b_{i}$ is the coefficient of the second OFDM signal $s_{b}(t)$, then $b_{i}=1-a_{i}$ for $i \in\{1,2$, $\ldots,(N-1) / 2,(N+3) / 2, \ldots, N\}$. We can note that the dc subcarrier of baseband signal is not used as in communication systems.

\section{Imaging Quality Parameters}

Three basic SAR parameters are commonly used to assess the imaging quality, i.e., the PSLR, the ISLR, and the image resolution $\delta_{r}$. The PSLR is given with [14]

$$
\operatorname{PSLR}=\frac{\max _{n}\left|y_{n}\right|^{2}}{\max _{k}\left|y_{k}\right|^{2}}
$$

and the ISLR is

$$
\operatorname{ISLR}=\frac{\sum_{n}\left|y_{n}\right|^{2}}{\sum_{k}\left|y_{k}\right|^{2}}
$$

where $\left|y_{n}\right|^{2}$ is the intensity of each pixel of the image outside the main lobe, and $\left|y_{k}\right|^{2}$ is the intensity of each pixel of the
TABLE I

MAIN PARAMETERS FOR SAR IMAge Simulation

\begin{tabular}{cc}
\hline \hline Parameter & Value \\
\hline Carrier frequency $f_{c}$ & $6 \mathrm{GHz}$ \\
Signal bandwidth $B_{r}$ & $20 \mathrm{MHz}$ \\
Pulse duration $T_{p}$ & $650 \mathrm{~ns}$ \\
Azimuth bandwidth $B_{a z}$ & $1300 \mathrm{~Hz}$ \\
Range resolution $\delta_{r}$ & $7.5 \mathrm{~m}$ \\
Azimuth resolution $\delta_{a z}$ & $2.7 \mathrm{~m}$ \\
\hline Altitude height $H$ & $798 \mathrm{~km}$ \\
Near incidence angle $\theta_{n}$ & $48.3^{\circ}$ \\
Far incidence angle $\theta_{f}$ & $50^{\circ}$ \\
\hline Non ambiguous PRF $F_{0}$ & $3530 \mathrm{~Hz}$ \\
Total slant swath width & $42.355 \mathrm{~km}$ \\
\hline Ambiguous PRF & $4060 \mathrm{~Hz}$ \\
Processing slant swath width & $36.8 \mathrm{~km}$ \\
\hline \hline
\end{tabular}

another parameter called $\Delta F$, which is based on the interambiguity function $A F^{s_{a}, s_{b}}\left(\tau, f_{d}\right)$ between $s_{a}(t)$ and $s_{b}(t)$ for the time delay $\tau$ and the frequency Doppler $f_{d}$ as follows:

$$
\Delta F=\frac{\max \left(A F^{s_{a}, s_{b}}\left(\tau, f_{d}\right)\right)}{A F^{s_{a}, s_{a}}(0,0)} .
$$

To reduce the range ambiguity, this last parameter must be as low as possible. In this paper, we propose to analyze the situation based on a coded-OFDM signal. The aim of this analysis is to optimize the OFDM signal regarding the four imaging quality parameters.

\section{Simulation Results}

\section{A. Configuration}

The parameters used for this simulation are listed in Table I and are mainly representative of spaceborne SAR system. It is worthwhile to note that a spaceborne system receives the echo from a specific pulse after several transmitted pulses due to the very long slant ranges involved. Thus, the sensing geometry is considered in order to take into account this effect. The PRF value of $3530 \mathrm{~Hz}$ corresponds to a nonambiguous case considering the geometric parameters described in Fig. 1, whereas the PRF value of $4060 \mathrm{~Hz}$ induces an ambiguous case. Thus, we consider a point target located at a slant range distance equal to $1271.468 \mathrm{~km}$ (e.g., $40 \mathrm{~km}$ in the SAR image), which means that this target is considered as a nonambiguous scattering point for SAR processing with $\mathrm{PRF}_{0}=3530 \mathrm{~Hz}$ but ambiguous with PRF $=4060 \mathrm{~Hz}$. In this ambiguous case, the SAR image presents a shadow around $3.2 \mathrm{~km}$ induced by the ambiguous position of the considered target point. To search over all the OFDM codes, we reduce the number of subcarriers to $N=13$ that induces the pulse duration of only $650 \mathrm{~ns}$. This value does not meet the classical SAR configuration, and for operational systems, the number of $N$ can increase to 513 or even higher values. Moreover, considering this configuration, the Doppler frequency is about $1300 \mathrm{~Hz}$. This value is around 1000 times lower than the subcarrier spacing. Under this assumption, the Doppler effect is neglected, and we can only focus on the correlation function $\Delta F_{0}$, which is the ambiguity 
TABLE II

PSLR, ISLR, $\delta_{r}$, AND $\Delta F_{0}$ FOR DIFFERENT CODED-OFDM SignAL SOLUTIONS

\begin{tabular}{c|ccccc}
\hline & $\begin{array}{c}\text { PSLR } \\
{[\mathrm{dB}]}\end{array}$ & $\begin{array}{c}\text { ISLR } \\
{[\mathrm{dB}]}\end{array}$ & $\begin{array}{c}\delta_{r} \\
{[\mathrm{~m}]}\end{array}$ & $\begin{array}{c}\Delta F_{0} \\
{[\mathrm{~dB}]}\end{array}$ & $\begin{array}{c}\text { Vector } \\
\left(a_{i}\right),\left(b_{i}\right)\end{array}$ \\
\hline \hline$s_{a 1}$ & -7.73 & -45.94 & 8.1 & -6.78 & {$[0110000111001]$} \\
$s_{b 1}$ & {$[1001110000110]$} \\
\hline$s_{a 2}$ & -7.79 & -45.04 & 8.1 & -7.74 & {$[0110110001001]$} \\
$s_{b 2}$ & {$[1001000110110]$} \\
\hline$s_{a 3}$ & -10.37 & -44.98 & 8.1 & -7.90 & {$[0111010010001]$} \\
$s_{b 3}$ & {$[1000100101110]$} \\
\hline Chirps & -12.35 & -43.53 & 7.5 & -5.75 & NONE \\
\hline $\begin{array}{c}\text { Full } \\
\text { Band }\end{array}$ & -12.19 & -49.89 & 7.5 & NONE & {$[111111011111]$} \\
\hline
\end{tabular}

\section{B. Results for the Coded-OFDM Signals}

As shown in Section II, we need to use two orthogonal signals or two signals with low cross correlation to suppress the range ambiguity. To simplify SAR imaging signal processing, both signals $s_{a}(t)$ and $s_{b}(t)$ must have the same characteristics, i.e., same carrier frequency, same subcarrier frequency $f_{i}$, and same bandwidth. The orthogonality is obtained by activating and turning off the different subcarriers of the OFDM signals. Furthermore, the total bandwidth available for the signals is divided between $s_{a}(t)$ and $s_{b}(t)$. The next step is to maximize the bandwidth of each signal to provide the same best range resolution for each signal, i.e., $\left(a_{i}\right)=\left[0,1, a_{3}, \ldots, a_{N-2}, 0,1\right]$. Taking into account the parameters previously defined, we can define a number of couples $\left(s_{a k}, s_{b k}\right)$ that resolve the range ambiguity. By computing all the different possible results for the eight subcarriers (from $i=3$ to $i=6$ and from $i=8$ to $i=$ 11) without the dc component (element $i=7$ ), only the three best couples $\left(s_{a k}, s_{b k}\right)$ are selected regarding the four imaging quality parameters defined in Section II-C. The different results of different couples are shown in Table II for the ambiguous PRF described in Table I. Moreover, we also compare the performances of these couples to the performances of the up- and down-chirp couples [4]. The PSLR values of the OFDM couples $\left(s_{a k}, s_{b k}\right)$ are higher than the PSLR values of the OFDM full band. This difference of PSLR values is due to the holes in the spectrum of the signals $s_{a}(t)$ and $s_{b}(t)$. The ISLR values of OFDM couples are higher than those of the OFDM full band but lower than the chirps' ISLR values. The range resolution value of the OFDM couples is close to the OFDM full band, which indicates that the holes in the spectrum have almost no influence on the resolution. Finally, the values of $\Delta F_{0}$ obtained for the OFDM couples are lower than the ones obtained with the up- and down-chirp couples. We can note that the different sequences of $\left(a_{i}\right)$ obtained exhibit a skew symmetry.

Fig. 2 shows the SAR images when entire signal processing is applied using the OFDM signal couple $\left(s_{a 3}, s_{b 3}\right)$, and Fig. 3 shows the SAR images in the case of using the chirp couple. As expected, the range ambiguity is better rejected in the case of OFDM signal that exhibits lower ISLR and $\Delta F_{0}$ than the chirp couple, and the levels of the shadow are reduced by $5 \mathrm{~dB}$. Moreover, the main scattering point is correctly imaged even if the impulse response of this point is better with the chirp couple
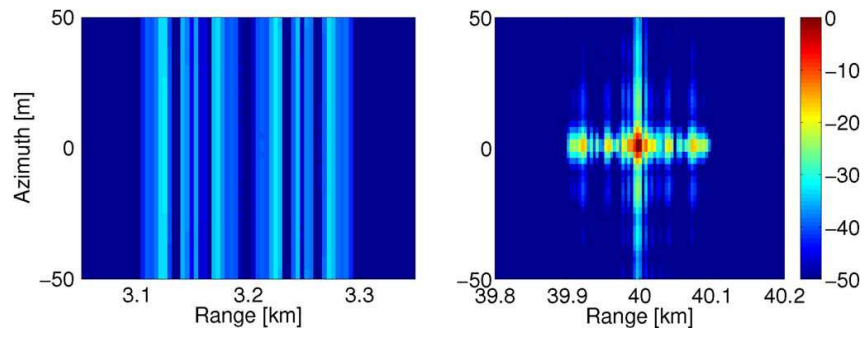

Fig. 2. SAR image with range ambiguity suppression procedure using OFDM signals $s_{a 3}(t)$ and $s_{b 3}(t)$. Focus on (left) the shadow and on (right) the image of the scattering point.
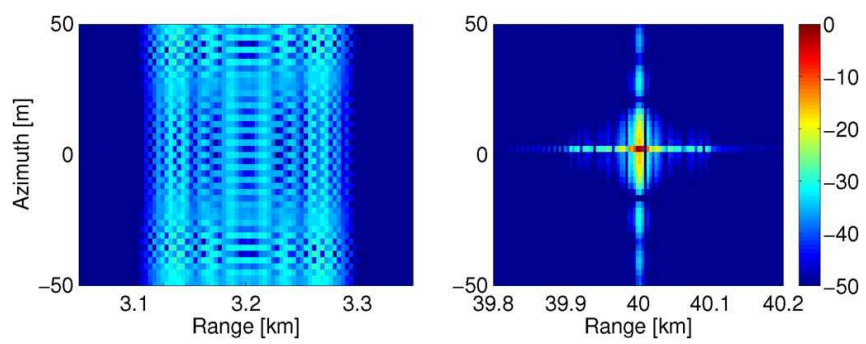

Fig. 3. SAR image with range ambiguity suppression procedure using up- and down-chirp couples. Focus on (left) the shadow and on (right) the image of the scattering point.

design for SAR image processing introduces a tradeoff between the quality of the impulse response of the scattering point and the rejection of the shadow of another scattering point located in the ambiguous part of the slant swath width.

\section{CONCLusion}

In this paper, we have demonstrated the possibility to reduce range ambiguities for a SAR image by using coded-OFDM signals. This reduction based on image quality parameters leads to multiple coded-OFDM signals in order to achieve the range ambiguity mitigation.

\section{REFERENCES}

[1] I. G. Cumming and F. H. Wong, Digital Processing of Synthetic Aperture Radar Data: Algorithms and Implementation. Boston, MA, USA: Artech House, 2005.

[2] J. Mittermayer, M. Younis, R. Metzig, S. Wollstadt, J. Martinez, and A. Meta, "Terra SAR-X system performance characterization and verification," IEEE Trans. Geosci. Remote Sens., vol. 48, no. 2, pp. 660-676, Feb. 2010 .

[3] A. Freeman, "On ambiguities in SAR design," in Proc. 6th Eur. Conf. Synthetic Aperture Radar, May 2006, pp. 1-4.

[4] J. Mittermayer and J. Martinez, "Analysis of range ambiguity suppression in SAR by up and down chirp modulation for point and distributed targets," in Proc. IEEE Int. Geosci. Remote Sens. Symp., Jul. 2003, vol. 6, pp. 4077-4079.

[5] J. Dall and A. Kusk, "Azimuth phase coding for range ambiguity suppression in SAR," in Proc. IEEE Int. Geosci. Remote Sens. Symp., Sep. 2004, vol. 3, pp. 1734-1737.

[6] J. Akhtar, "Cancellation of range ambiguities with block coding techniques," in Proc. IEEE Radar Conf., May 2009, pp. 1-6.

[7] W.-Q. Wang, "Mitigating range ambiguities in high-PRF SAR with OFDM waveform diversity," IEEE Geosci. Remote Sens. Lett., vol. 10, no. 1, pp. 101-105, Jan. 2013.

[8] J. Schuerger and D. Garmatyuk, "Deception jamming modeling in radar sensor networks," in Proc. IEEE Mil. Commun. Conf., 2008, pp. 1-7.

「91 M. Sebt, A. Sheikhi, and M. Nayebi, "Orthogonal frequency-division 
low peak-to-average power ratio," IET Radar, Sonar Navig., vol. 3, no. 2, pp. 122-132, Apr. 2009.

[10] S. Sen and A. Nehorai, "Adaptive design of OFDM radar signal with improved wideband ambiguity function," IEEE Trans. Signal Process., vol. 58, no. 2, pp. 928-933, Feb. 2010.

[11] J. C. Curlander and R. N. McDonough, Synthetic Aperture RadarSystems and Signal Processing. New York, NY, USA: Wiley, 1991.

[12] V. Riché, S. Méric, and E. Pottier, "Range ambiguity suppression in an OFDM SAR configuration," in Proc. 9th Eur. Conf. Synthetic Aperture Radar, Apr. 2012, pp. 115-118.

[13] H. Sari, G. Karam, and I. Jeanclaude, "Transmission techniques for digital terrestrial TV broadcasting," IEEE Commun. Mag., vol. 33, no. 2, pp. 100-109, Feb. 1995.

[14] R. Davis, R. Fante, and R. Perry, "Phase-coded waveforms for radar," IEEE Trans. Aerosp. Electron. Syst., vol. 43, no. 1, pp. 401-408, Jan. 2007.

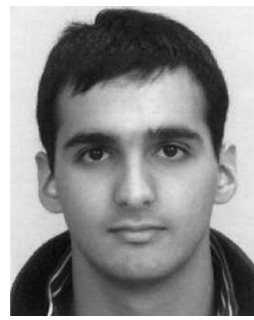

waveform).
Vishal Riché received the M.S. degree in signal and circuit from the Université de Bretagne Occidentale, Brest, France, in 2009. He is pursuing the Ph.D. degree in electronics in the Institute of Electronics and Telecommunications of Rennes, Rennes, France, working on radar system dedicated to specific SAR applications (remote sensing, MIMO configuration). $\mathrm{He}$ is currently with the Fraunhoffer Institut, Wachtberg, Germany and is interested in the application of nonconventional waveform for radar application (orthogonal frequency-division multiplexing

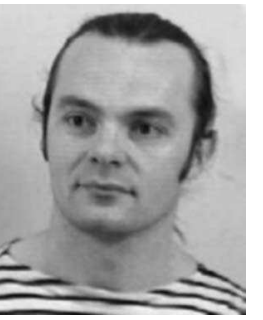

Stéphane Méric (M’08) simultaneously received the Electrical Engineer Diploma from the National Institute for Applied Sciences of Rennes (INSA), Rennes, France, and the M.S. degree in signal processing and telecommunications from the University of Rennes 1, Rennes, in 1991 and the Ph.D. degree in electronics from INSA in 1996.

Since 2000, he has been an Assistant Professor with INSA, and in 2005, he joined the SAPHIR Team, Institute of Electronics and Telecommunications of Rennes (IETR, CNRS UMR 6164), Rennes.

$\mathrm{He}$ is interested in using SAR data in radargrammetric applications. Furthermore, he is currently working on radar system (i.e., $\mathrm{CW}$ and $\mathrm{FMCW}$ ) dedicated to specific SAR applications (i.e., radar imaging in motorway context, remote sensing, MIMO configuration, and passive radar imaging). His education activities are about analog electronics, signal processing, radar and radar imaging, and electromagnetic diffraction.

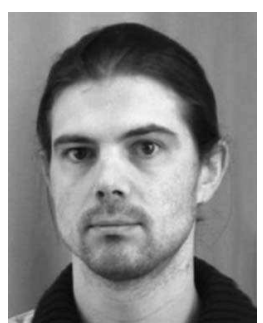

Jean-Yves Baudais received the M.Sc. degree and the $\mathrm{Ph} . \mathrm{D}$. degree in electrical engineering from the National Institute for Applied Sciences of Rennes (INSA), Rennes, France, in 1997 and 2001, respectively.

In 2002, he joined the French National Centre for Scientific Research (CNRS), Paris, France, where he is currently a Researcher with the Digital Communication Systems Team (SCN), Institute of Electronics and Telecommunications of Rennes (IETR), Rennes. $\mathrm{He}$ is currently the Head of the SCN Team. His general interests are in the areas of signal processing and digital communications. His current research focuses on transmitter design and receiver diversity techniques for multiuser and multicarrier communication, including space-time coding.

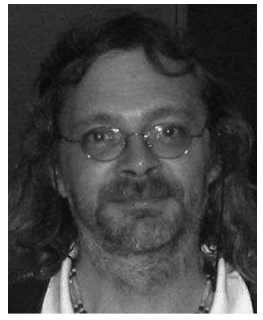

Éric Pottier (M'95-SM'06-F'11) received the M.Sc. and Ph.D. degrees in signal processing and telecommunication from the University of Rennes 1, Rennes, France, in 1987 and 1990, respectively, and the Habilitation from the University of Nantes, Nantes, France, in 1998.

Since 1999, he has been a Full Professor with the University of Rennes 1, where he is currently the Deputy Director of the Institute of Electronics and Telecommunications of Rennes (IETR, CNRS UMR 6164), Rennes. He is also the Head of the Image and Remote Sensing Group, SAPHIR Team. Since 1989, he has supervised more than 30 research students to graduation (Ph.D.) in radar polarimetry covering areas from theory to remote sensing applications. He has been invited to present 46 presentations in international conferences. He has published a book coauthored with Dr. J. S. Lee in 2009 , nine publications in books, and more than 50 papers in refereed journals, and he presented more than 330 papers during international conferences, symposiums, and workshops. He has presented advance courses and seminars on radar polarimetry to a wide range of organizations (i.e., DLR, NASDA, JRC, RESTEC, and IECAS) and events (i.e., ISAP2000, EUSAR04-06-10, NATO-04-06, PolInSAR05-11, JAXA06, and IGARSS03-05-07-08-09-10). His current activities of research and education are centered in the topics of analog electronics, microwave theory, and radar imaging with emphasis in radar polarimetry. His research covers a wide spectrum of areas from radar image processing (e.g., SAR and ISAR), polarimetric scattering modeling, supervised/unsupervised polarimetric segmentation, and classification to fundamentals and basic theory of polarimetry.

Prof. Pottier has chaired and organized 35 sessions in international conferences and was a member of the Technical and Scientific committees of 32 international symposiums or conferences. He was the recipient of the Best Paper Award at the Third European Conference on Synthetic Aperture Radar (EUSAR2000), the 2007 IEEE GRS-S Letters Prize Paper Award, and the 2007 IEEE GRS-S Education Award "In recognition of his significant educational contributions to Geoscience and Remote Sensing." 